\title{
Detección de deficiencias de anticuerpos y caracterización clínico-demográfica en pacientes pediátricos con enfermedades alérgicas
}

\section{Detection of antibody deficiencies and clinical-demographic characterization in pediatric patients with allergic diseases}

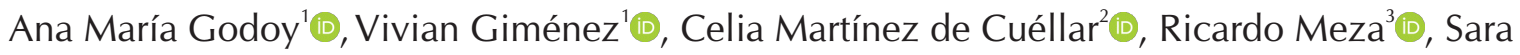

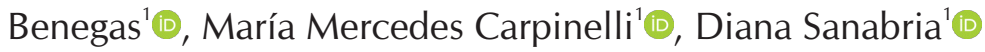 \\ ${ }^{1}$ Universidad Nacional de Asunción, Instituto de Investigaciones en Ciencias de la Salud, Departamento \\ de Inmunología. San Lorenzo, Paraguay. \\ ${ }^{2}$ Ministerio de Salud Pública y Bienestar Social, Instituto de Medicina Tropical, Centro Nacional de \\ Referencia de Inmunodeficiencias Primarias. Asunción, Paraguay. \\ ${ }^{3}$ Universidad Nacional de Asunción, Facultad de Ciencias Médicas, Unidad Pediátrica Ambulatoria de la \\ Especialidad Asma, Alergia e Inmunología del Hospital de Clínicas. San Lorenzo, Paraguay.
}

\section{RESUMEN}

Introducción: Las deficiencias predominantes de anticuerpos (DPA) cursan con disminución de niveles séricos de inmunoglobulinas (hipogammaglobulinemia), infecciones recurrentes y se ha reportado su asociación con las alergias. La información sobre su frecuencia en niños alérgicos es limitada y en Paraguay no existen datos al respecto. Objetivo: Detectar DPA y establecer su frecuencia en pacientes pediátricos con enfermedades alérgicas atendidos en un hospital de referencia del país. Materiales y métodos: Fueron evaluados 64 pacientes pediátricos (1 a 17 años de edad) con diagnóstico de alergia, atendidos en la Unidad Pediátrica Ambulatoria-Especialidad Asma, Alergia e Inmunología del Hospital de Clínicas (periodo 2018-2019). Se midieron los niveles séricos de IgA, IgG e IgM por el método de inmunodifusión radial y se aplicaron criterios de diagnóstico fenotípico a los casos de hipogammaglobulinemia para definir la DPA. Resultados: La mediana de edad fue de 5 años (RIQ: 2 - 8), con predominio del sexo masculino (58\%). Las alergias más frecuentes fueron asma (38\%) y rinitis (34\%), además predominaron las infecciones respiratorias recurrentes $(80 \%)$. La frecuencia de DPA fue de $17 \%(11 / 64)$, detectándose 6 casos de deficiencia de inmunoglobulina $\mathrm{A}$,

\section{ABSTRACT}

Introduction: Predominant antibody deficiencies (PAD) present with decreased serum levels of immunoglobulins (hypogammaglobulinemia), recurrent infections and their association with allergies has been reported. Information on its frequency in allergic children is limited and in Paraguay there are no data in this regard. Objective: To detect PAD and establish its frequency in pediatric patients with allergic diseases treated at a reference hospital in the country. Materials and methods: 64 pediatric patients ( 1 to 17 years of age) with a diagnosis of allergy, treated in the Pediatric Outpatient Unit-Specialty Asthma, Allergy and Immunology of the Hospital de Clínicas (from 2018 to 2019) were evaluated. Serum levels of IgA, IgG and IgM were measured by the radial immunodiffusion method and phenotypic diagnostic criteria were applied to hypogammaglobulinemia cases to define PAD. Results: The median age was 5 years (IQR: 2 $8)$, with a predominance of males $(58 \%)$. The most frequent allergies were asthma (38\%) and rhinitis (34\%), and recurrent respiratory infections $(80 \%)$ predominated. The frequency of PAD was $17 \%(11 / 64)$, with 6 cases of immunoglobulin A deficiency detected, 4 isolated IgG deficiencies and a common variable immunodeficiency

Correspondencia: Diana Sanabria, correo: dsanabria@iics.una.py

Conflicto de interés: Los autores declaran no poseer conflicto de interés

Fuente de financiamiento: La realización de este estudio fue posible gracias al financiamiento otorgado por los fondos para investigación del Rectorado de la Universidad Nacional de Asunción en su convocatoria del año 2018.

Recibido: 17/09/2021 Aceptado: 10/11/2021

DOI: https://doi.org/10.31698/ped.48032021008

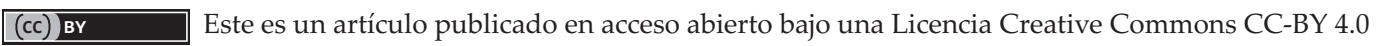


4 deficiencias aisladas de IgG y una inmunodeficiencia común variable. No se observaron diferencias significativas al comparar características clínicodemográficas entre pacientes alérgicos con y sin DPA. Conclusiones: La frecuencia de DPA fue elevada, por lo que se sugiere considerar el estudio de inmunoglobulinas séricas en pacientes pediátricos con enfermedades alérgicas para una detección y tratamiento oportunos.

Palabras clave: Enfermedades de Inmunodeficiencia Primaria; Síndrome de Deficiencia de Anticuerpos; Alergia; Pediatría.

\section{INTRODUCCIÓN}

Las inmunodeficiencias primarias (IDPs) son un grupo heterogéneo de trastornos genéticos poco frecuentes que afectan la función del sistema inmune, caracterizándose por infecciones recurrentes, severas y oportunistas $^{(1)}$. Las deficiencias predominantes de anticuerpos (DPA) constituyen más del 50\% de las IDPs, resultan de una alteración génica en el desarrollo, maduración y/o función de células B, siendo su principal manifestación clínica las infecciones respiratorias recurrentes $y$, la característica inmunológica más resaltante es la disminución de los niveles séricos de IgA, IgG y/o IgM por debajo del rango considerado normal

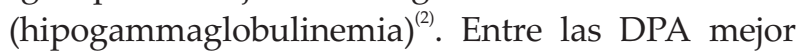
descritas están la deficiencia de inmunoglobulina A (DIgA), inmunodeficiencia común variable (CVID), la deficiencia de anticuerpos específicos (SAD), agammaglobulinemia ligada al X (XLA) y las deficiencias de subclases de IgG. Además de las infecciones recurrentes en las DPA, se reportó su asociación con las alergias ${ }^{(3)}$.

Las sociedades de todo el mundo se enfrentan a un riesgo progresivamente incrementado de problemas de salud mediados por el sistema inmune, tales como las alergias, cuyas frecuencias son cada vez mayores, representando las patologías crónicas más comunes en niños, con una prevalencia de 7,3\% (eczema) a 14,6\% (asma y rinitis) en niños de entre 13 y 14 años de edad, y en el rango de 6 a 7 años, la prevalencia oscila entre $7,9 \%$ (eczema) a $11,7 \%$ para el asma ${ }^{(4)}$. Las alergias pueden afectar seriamente la were also detected. No significant differences were observed when comparing clinical-demographic characteristics between allergic patients with and without PAD. Conclusions: The frequency of PAD was high, so we suggest considering serum immunoglobulins studies in pediatric patients with allergic diseases for timely detection and treatment.

Keywords: Primary Immunodeficiency Diseases; Antibody Deficiency Syndrome; Allergy; Pediatrics.

salud física y psicológica de quienes las padecen, ejerciendo una importante carga en la calidad de vida de los afectados y de la sociedad que los rodea ${ }^{(5)}$. En la literatura científica se encontraron varios reportes sobre la prevalencia de las alergias en individuos afectados por DPA; Shkalim et al. ${ }^{(6)}$ reportaron una prevalencia de $22 \%$ de enfermedad alérgica en niños con DIgA. Por otra parte, Ramírez et al. ${ }^{(7)}$ evaluaron a 52 pacientes con CVID y encontraron que el $25 \%$ eran alérgicos. Sin embargo, es limitada la información sobre la frecuencia de DPA en individuos alérgicos, ${ }^{(8,9)}$ Costa-Carvalho et al. ${ }^{(10)}$ observaron una frecuencia de $7 \%(2 / 27)$ de deficiencias de anticuerpos en niños con alergias respiratorias. Además, Yuruker et al. ${ }^{(11)}$ evaluaron a 125 niños con asma, observando que 76/125 (60,8\%) tenía una deficiencia de anticuerpos.

En Paraguay, la situación de las IDPs en general es que probablemente existe un subdiagnóstico y subregistro de casos, por motivos como la baja sospecha clínica y el difícil acceso a las pruebas diagnósticas, se suma a esto, la escasa producción científica local sobre estas patologías. En cuanto a las DPA, las investigaciones realizadas en el país se limitan a la evaluación de pacientes con infecciones recurrentes, ${ }^{(12)}$ no existiendo aún información sobre la prevalencia de estas deficiencias en población pediátrica con alergias. El objetivo de este estudio fue detectar deficiencias de anticuerpos y establecer su frecuencia en pacientes pediátricos con alergias atendidos en un hospital de referencia, además de 
evaluar el comportamiento de características clínicodemográficas según la presencia de una DPA en los pacientes alérgicos evaluados.

\section{MATERIALES Y MÉTODOS}

\section{Tipo de estudio}

El diseño fue observacional, descriptivo, de corte transversal, y de carácter prospectivo en cuanto a la toma de muestra y realización de las mediciones en los sujetos de estudio.

\section{Pacientes y criterios de selección}

Se estudió a 64 pacientes pediátricos, de 1 a 17 años de edad, de ambos sexos y con diagnóstico de alergia, atendidos en consulta ambulatoria de un hospital de referencia del país, desde julio de 2018 hasta mayo de 2019.

El muestreo fue no probabilístico por conveniencia, identificándose inicialmente a 80 pacientes con diagnóstico de enfermedad alérgica (asma, rinitis alérgica, alergia a alimentos y/o dermatitis atópica) establecido por un médico especialista de la Unidad Pediátrica Ambulatoria-Especialidad Asma, Alergia e Inmunología del Hospital de Clínicas (Facultad de Ciencias Médicas-Universidad Nacional de Asunción). De este total, fueron excluidos 13 pacientes cuyos responsables no aceptaron su participación, además de 2 pacientes con hipogamaglobulinemia y un paciente con patología hemato-oncológica, según constaba en la historia clínica u otro documento del paciente, lo que fue constatado al momento de la consulta médica. Finalmente, cada uno de los 64 pacientes seleccionados fue remitido al Departamento de Inmunología del Instituto de Investigaciones en Ciencias de la Salud (IICS) siguientes pasos del estudio.

\section{Determinación de los niveles séricos de inmuno- globulinas totales}

Se extrajo a cada paciente $3 \mathrm{~mL}$ de sangre por punción venosa, de la cual se obtuvo suero, donde se midieron las concentraciones de inmunoglobulinas $\operatorname{Ig} \mathrm{A}, \operatorname{Ig} \mathrm{G}$ e $\operatorname{Ig} \mathrm{M}$, en $\mathrm{mg} / \mathrm{dL}$, por el método de inmunodifusión radial (IDR), en placas comerciales (Biocientífica S.A, Buenos Aires, Argentina) y siguiendo las instrucciones del fabricante. La concentración sérica de $\operatorname{IgE}$ total $(\mathrm{UI} / \mathrm{mL})$ se determinó mediante un kit comercial de ELISA (Human, Alemania). Para todas las inmunoglobulinas se consideraron niveles séricos normales cuando resultaron dentro de los rangos de referencia, según edad, establecidos por los fabricantes de los reactivos utilizados.

\section{Detección de deficiencias predominantes de anticuerpos (DPA)}

Se identificaron los casos de hipogammaglobulinemia, definida como la disminución por debajo del rango de referencia de al menos un isotipo $\operatorname{IgA}$, IgG y/o IgM. Para la IgA se consideró un valor inferior a $7 \mathrm{mg} / \mathrm{dL}$ para establecer una deficiencia total de $\mathrm{IgA}$, y cuando resultó mayor a $7 \mathrm{mg} / \mathrm{dL}$ pero inferior al rango de referencia, correspondió a una deficiencia parcial de $\operatorname{IgA}{ }^{(13)}$. Para la $\operatorname{IgG}$ e $\operatorname{IgM}$ se consideró una deficiencia cuando el valor resultó menor al límite inferior del rango de referencia para la edad ${ }^{(2)}$. A cada paciente que presentó hipogammaglobulinemia, se le extrajo una nueva muestra de sangre 4 semanas después de la primera, para verificar el resultado. Seguidamente, se aplicó el algoritmo de diagnóstico fenotípico correspondiente a DPA, reportado por la Unión Internacional de Sociedades de Inmunología (IUIS) ${ }^{(14)}$, de modo a definir la deficiencia de anticuerpo para cada paciente con hipogammaglobulinemia, además, se tuvieron en cuenta los criterios diagnósticos de la Academia Americana de Alergia, Asma e Inmunología-AAAAI ${ }^{(15)}$.

\section{Caracterización clínico-demográfica de la pobla- ción de estudio}

Mediante la aplicación de un cuestionario de investigación se evaluaron características demográficas como edad, sexo, procedencia, situación educativa, y características clínicas como el tipo de alergia, edad de inicio y frecuencia de exacerbaciones (veces/año que el episodio alérgico requirió atención en urgencias u hospitalización), también se indagó sobre la presencia de infecciones recurrentes (más de 2 veces/año), el tratamiento de las alergias y los antecedentes familiares. Se evaluó el comportamiento de características clínicodemográficas según la presencia de una deficiencia de anticuerpos, estratificando a la población de estudio en grupos de pacientes alérgicos con y sin DPA. 


\section{Asuntos éticos}

Los responsables de los menores de edad firmaron un consentimiento informado autorizando su participación en este estudio. Se respetó en todo momento la confidencialidad de los datos de pacientes y también de sus responsables. Los casos definidos como una DPA se comunicaron debidamente al responsable del niño y a su médico tratante, derivando al paciente con un médico especialista en IDPs (Consultorio de Inmunodeficiencias Primarias-Servicio de Pediatría del Instituto de Medicina Tropical - MSPyBS) para el establecimiento de un diagnóstico y tratamiento. El protocolo de investigación fue aprobado por el Comité de Ética en Investigación del IICS (código P07/2017).

\section{Análisis estadísticos}

Los datos fueron analizados con el programa estadístico Epi $\mathrm{Info}^{\mathrm{TM}}$ versión 7.2 para Windows (CDC-Centro para el control y la prevención de enfermedades). Las variables categóricas se describieron mediante frecuencias (n) y porcentajes (\%), mientras que las cuantitativas se presentaron con medianas y rangos intercuartílicos (RIQ). Se realizaron comparaciones estadísticas de características clínico-demográficas entre los grupos de pacientes con y sin DPA, así, para comparar variables continuas se utilizó la prueba de MannWhitney y para variables cualitativas se empleó la prueba exacta de Fisher $(\mathrm{p}<0,05)$.

\section{RESULTADOS}

En este estudio fueron evaluados 64 pacientes pediátricos con diagnóstico de alergia, observándose que la mediana de edad fue de 5 años (RIQ: 2 - 8) y predominaron los participantes del sexo masculino (58\%). En cuanto a la procedencia de los pacientes, la mayoría eran de ciudades del Departamento Central (56\%) y de la ciudad de Asunción (20\%). Por otra parte, 40 pacientes pediátricos se encontraban en edad de asistir a una institución educativa, encontrándose que un $22 \%$ de ellos asistían de forma irregular (ausencias frecuentes) por repeticiones de los episodios alérgicos, exacerbaciones de los mismos y/o recurrencia de infecciones (Tabla 1 ).
En la caracterización clínica de los pacientes evaluados (Tabla 1) se observó que los tipos de alergias más frecuentes fueron el asma $(38 \%)$ y la rinitis $(34 \%)$, siendo que la edad de inicio de las alergias presentó una mediana de 1 año (RIQ: 0,5 - 3) $y$, para la frecuencia de exacerbaciones de los episodios alérgicos, la mediana fue de 4 veces/año (RIQ: 2 - 5). Por otra parte, el 83\% de los pacientes presentó al menos un familiar con enfermedad alérgica, predominando los de primer grado de consanguinidad. Un $73 \%$ recibía tratamiento antialérgico al momento del estudio, y solo en 6 pacientes se observó la realización previa de la prueba de IgE específica para alérgeno (RAST), la que resultó positiva para ácaros (2/6), polvo de casa (1/6) y a proteínas de leche de vaca (2/6). Además, en el $92 \%$ de los pacientes se observó la presencia de infecciones a repetición (mayor a 2 veces /año).

Los niveles séricos de inmunoglubulinas totales se determinaron en la población evaluada de pacientes pediátricos con enfermedades alérgicas. Una proporción de 53/64 (83\%) pacientes presentaron niveles de IgA, IgG e IgM en el rango considerado normal o de referencia para la edad. En cuanto a la medición de IgE, los niveles séricos resultaron superiores al rango de referencia en 56/64 (88\%) de los pacientes evaluados. Los datos estadísticos correspondientes al estudio de inmunoglobulinas séricas en la población total evaluada se presentan en la Tabla 2.

Si bien en la mayoría de los pacientes se observó niveles séricos normales de inmunoglobulinas totales, en 11/64 (17\%) de los pacientes alérgicos se detectó una hipogammaglobulinemia de al menos un isotipo de inmunoglobulina (IgA, IgG y/o IgM). Para cada uno de estos casos se definió la deficiencia de anticuerpo más probable, clasificándolas según el algoritmo de diagnóstico fenotípico reportado por el grupo de expertos IUIS, detectándose así las siguientes DPA: 6 casos de deficiencia de $\operatorname{IgA}$ (pacientes 2, 5, 10, 20, 27 y 58), 4 casos de deficiencia aislada de IgG (pacientes 21, 48, 60 y 62) y un caso de inmunodeficiencia común variable (paciente 6). En este grupo de pacientes alérgicos con DPA se observó una mediana de 7 años de edad (RIQ: 3 - 10 años), con un ligero predominio del sexo femenino $(55 \%)$. La rinitis alérgica $(45 \%)$ y el asma $(36 \%)$ 
fueron las alergias predominantes, y en cuanto a la edad de inicio de los episodios alérgicos, se observó una mediana de 1 año (RIQ: 0,5 - 4), la mediana para la frecuencia de exacerbaciones de estos episodios fue de 2 veces/año (RIQ: 2 - 4). El 73\% tenía familiares de primer grado de consanguinidad (padre y/o madre) con enfermedad alérgica, además, el $73 \%$ de los pacientes se encontraban con tratamiento para la alergia. En cuanto a la presentación de infecciones recurrentes, en el 100\% de los niños alérgicos con DPA se observó presencia de cuadros infecciosos a repetición, de estas las más frecuentes fueron las infecciones del tracto respiratorio $(72 \%)$. La caracterización de pecientes alérgicos con DPA se presenta en la Tabla 3.

Tabla 1. Caracterización clínico-demográfica de pacientes pediátricos con enfermedades alérgicas. (n=64)

\begin{tabular}{|c|c|c|}
\hline Característica & $\mathbf{n}$ & $\%$ \\
\hline \multicolumn{3}{|l|}{ Sexo } \\
\hline Masculino & 37 & 58 \\
\hline Femenino & 27 & 42 \\
\hline \multicolumn{3}{|l|}{ Procedencia } \\
\hline Asunción & 13 & 20 \\
\hline Departamento Central & 36 & 56 \\
\hline Ciudades del interior & 15 & 24 \\
\hline \multicolumn{3}{|l|}{ Situación escolar $(n=40)^{a}$} \\
\hline Asiste regularmente & 29 & 73 \\
\hline Asiste de forma irregular ${ }^{b}$ & 9 & 22 \\
\hline Abandono & 2 & 5 \\
\hline \multicolumn{3}{|l|}{ Tipos de alergias } \\
\hline Asma & 24 & 38 \\
\hline Rinitis alérgica & 22 & 34 \\
\hline Dermatitis atópica y/o AA & 10 & 16 \\
\hline Más de un tipo de alergia & 8 & 12 \\
\hline \multicolumn{3}{|l|}{ Antecedente familiar de alergia } \\
\hline Fliar. de 1 er grado (padre y/o madre) & 36 & 56 \\
\hline Fliar. de 2do grado (hno/a y/o abuelo/a) & 12 & 19 \\
\hline Fliar. de 3er grado (tío/a y/o bisabuelo/a) & 5 & 8 \\
\hline \multicolumn{3}{|l|}{ Presencia de infección recurrente } \\
\hline ITRI (neumonías y/o bronquitis) & 29 & 46 \\
\hline ITRS (amigdalitis y/o faringitis) & 22 & 34 \\
\hline Fiebre sin foco (o etiología determinada) & 2 & 3 \\
\hline Parotiditis & 2 & 3 \\
\hline Otras infecciones ${ }^{c}$ & 4 & 6 \\
\hline \multicolumn{3}{|l|}{ Tratamiento antialérgico } \\
\hline $\mathrm{Si}$ & 47 & 73 \\
\hline No & 17 & 27 \\
\hline
\end{tabular}

Tabla 2.Niveles séricos de inmunoglobulinas en la población total estudiada de pacientes pediátricos con alergias. $(n=64)$

\begin{tabular}{lccc}
\hline Determinación & Mediana & RIQ & Rango total \\
\hline $\operatorname{IgA}(\mathrm{mg} / \mathrm{dL})$ & 91,5 & $48,2-131,8$ & $0,6-269,0$ \\
$\operatorname{lgG}(\mathrm{mg} / \mathrm{dL})$ & 809,9 & $589,3-944,5$ & $316,2-2464,0$ \\
$\operatorname{lgM}(\mathrm{mg} / \mathrm{dL})$ & 146,2 & $102,2-177,3$ & $38,6-442,0$ \\
$\operatorname{IgE}(\mathrm{UI} / \mathrm{mL})$ & 133,0 & $56,3-544,0$ & $2,7-984,3$ \\
\hline
\end{tabular}

Ig: inmunoglobulina, mg/dL: miligramos por decilitros, Ul/mL: unidades internacionales por mililitros, RIQ: rango intercuartílico. 
Tabla 3. Caracterización de pacientes pediátricos alérgicos con deficiencias de anticuerpos. $(\mathrm{n}=11)$

\begin{tabular}{|c|c|c|c|c|c|c|c|c|c|}
\hline ID & Sexo & Edad $^{a}$ & $\lg \mathrm{A}(\mathrm{mg} / \mathrm{dL})$ & $\operatorname{lgG}(\mathrm{mg} / \mathrm{dL})$ & $\operatorname{lgM}(\mathrm{mg} / \mathrm{dL})$ & $\operatorname{lgE}(\mathrm{UI} / \mathrm{mL})$ & Tipo de alergia & Tipo de IR & DPA \\
\hline 2 & $M$ & 4 & 16,9 & 841,8 & 159,7 & 27,3 & RA & Amigdalitis & DIgA \\
\hline 5 & $\mathrm{~F}$ & 17 & 0,6 & 2464,0 & 442,0 & 546,8 & $\mathrm{DA}$ & Cutánea & DIgA \\
\hline 6 & $\mathrm{~F}$ & 7 & 8,6 & 316,2 & 47,4 & 5,0 & RA & Neumonía & CVID \\
\hline 10 & $\mathrm{~F}$ & 11 & 58,4 & 789,1 & 90,4 & 121,4 & RA & Candidiasis & DIgA \\
\hline 20 & $\mathrm{~F}$ & 10 & 0,6 & 1360,5 & 114,1 & 79,8 & Asma & Parotiditis & DIgA \\
\hline 21 & M & 8 & 67,7 & 402,9 & 38,6 & 97,3 & Asma & Amigdalitis & DIgG \\
\hline 27 & M & 1 & 7,8 & 402,9 & 38,6 & 556,9 & Asma & Neumonía & DIgA \\
\hline 48 & $M$ & 3 & 109,0 & 447,7 & 152,9 & 618,6 & RA & Bronquitis & DIgG \\
\hline 58 & $\mathrm{~F}$ & 2 & 16,3 & 540,3 & 102,1 & 13,7 & $\mathrm{AA}$ & Amigdalitis & DIgA \\
\hline 60 & $\mathrm{~F}$ & 8 & 94,2 & 430,6 & 228,5 & 880,4 & Asma & Bronquitis & DIgG \\
\hline 62 & M & 5 & 86 & 385,2 & 109,0 & 439,0 & RA & Faringitis & DIgG \\
\hline
\end{tabular}

ID: código de identificación del paciente, a: en años, M: masculino, F: femenino, RA: rinitis alérgica, DA: dermatitis atópica, AA: alergia a alimentos, IR: infección recurrente, DIgA: deficiencia de IgA, CVID: inmunodeficiencia común variable, DIgG: deficiencia aislada de IgG. En negrita, los valores de inmunoglobulinas con niveles séricos disminuidos.

Finalmente, en busca de alguna característica distintiva entre el grupo de pacientes alérgicos con DPA $(n=11)$ y sin DPA $(n=53)$, se evaluó el comportamiento clínico-demográfico entre ambos grupos, no observándose diferencias estadísticamente significativas en las características comparadas, a excepción de los niveles séricos de $\operatorname{Ig} \mathrm{A}$ e $\operatorname{IgG}$ que fueron significativamente más bajos en los pacientes con DPA (Tabla 4), comportamiento esperado en estos pacientes con hipogamaglobulinemia, lo que condujo a clasificarlos como casos de DPA detectadas en este estudio.

Tabla 4. Comportamiento clínico-demográfico en pacientes pediátricos con alergias según la presencia de DPA. $(n=64)$

\begin{tabular}{|c|c|c|c|}
\hline Característica & $\begin{array}{l}\text { Pacientes alérgicos } \\
\text { sin DPA }(n=53)\end{array}$ & $\begin{array}{c}\text { Pacientes alérgicos } \\
\text { con DPA }(n=11)\end{array}$ & $\begin{array}{c}\text { Valor } \\
\mathbf{p}^{\mathrm{b}}\end{array}$ \\
\hline Edad al momento del estudio $^{a}$ & $4(2-6)$ & $7(3-10)$ & 0,233 \\
\hline Sexo (Femenino/Masculino), \% & $40 / 60$ & $55 / 45$ & 0,505 \\
\hline Edad de inicio de alergias ${ }^{\mathrm{a}}$ & $1(0,6-3)$ & $1(0,5-4)$ & 0,993 \\
\hline Presencia de asma, \% (n) & $49(26)$ & $36(4)$ & 0,520 \\
\hline Presencia de rinitis alérgica, \% (n) & $60(32)$ & $55(6)$ & 0,746 \\
\hline Infecciones recurrentes del TRI, \% (n) & $47(25)$ & $36(4)$ & 1,000 \\
\hline Infecciones recurrentes del TRS, \% (n) & $34(18)$ & $36(4)$ & 0,568 \\
\hline Familiar de primer grado con alergia, \% (n) & $52(28)$ & $73(8)$ & 0,322 \\
\hline Exacerbaciones de alergias (veces/año) ${ }^{a}$ & $4(3-6)$ & $2(2-4)$ & 0,078 \\
\hline Niveles séricos de $\operatorname{lgA}(\mathrm{UI} / \mathrm{mL})^{\mathrm{a}}$ & $101,4(57,4-138,4)$ & $16,9(7,8-86,0)$ & $\mathbf{0 , 0 0 1}$ \\
\hline Niveles séricos de $\operatorname{lgG}(\mathrm{UI} / \mathrm{mL})^{\mathrm{a}}$ & $841,8(673,0-948,1)$ & $447,7(402,9-841,8)$ & $\mathbf{0 , 0 2 0}$ \\
\hline Niveles séricos de $\lg M(\mathrm{UI} / \mathrm{mL})^{\mathrm{a}}$ & $147,7(120,3-180,8)$ & $109,0(47,4-159,7)$ & 0,126 \\
\hline Niveles séricos de $\operatorname{lgE}(\mathrm{UI} / \mathrm{mL})^{\mathrm{a}}$ & $135,7(56,4-462,5)$ & $121,4(27,3-556,9)$ & 0,992 \\
\hline
\end{tabular}

${ }^{a}$ : expresado en mediana y RIQ, TRI: tracto respiratorio inferior, TRS: Tracto respiratorio superior, ${ }^{\text {b }}$ : obtenidos mediante la prueba de Mann-Whitney para variables cuantitativas y por la prueba exacta de Fisher para variables cualitativas (nivel de significancia estadística: $p<0,05)$.

\section{DISCUSIÓN}

En este estudio evaluamos a 64 pacientes pediátricos alérgicos y observamos una mediana de edad de 5 años con predominio del sexo masculino; se reportaron resultados muy similares en cuanto a edad y sexo en series de niños con rinitis alérgica ${ }^{(16)}$, además, está documentado que el asma predomina en varones durante la infancia ${ }^{(17)}$. Por otra parte, la mayoría de los pacientes eran de ciudades del Departamento Central y de Asunción; la escasa afluencia de pacientes de ciudades del interior 
podría deberse a la incorporación de la especialidad de alergia en varios hospitales regionales. También se observó que 9/40 de los niños en edad escolar presentaron ausentismo frecuente; en niños alérgicos son frecuentes las infecciones respiratorias recurrentes, debido a la adherencia aumentada de patógenos al epitelio respiratorio inflamado, las que serían razones de ausencias en la escuela, además, un estudio en niños con rinitis alérgica menciona que esta afección puede comprometer la calidad de vida alterando su desempeño escolar ${ }^{(18)}$.

Las alergias más frecuentes en este estudio fueron el asma y la rinitis, de hecho, la forma clínica de presentación más frecuente de las alergias es la respiratoria ${ }^{(19)}$. Con respecto a los antecedentes familiares, más de la mitad de los niños tenía padre y/o madre con enfermedad alérgica; un estudio en niños con rinitis alérgica mostró que el $68 \%$ tenían padres alérgicos ${ }^{(16)}$. Por otra parte, un $27 \%$ de los pacientes no recibía tratamiento para la alergia al momento del estudio; las alergias no tratadas adecuadamente pueden contribuir a la exacerbación de los episodios alérgicos y a la presentación de infecciones con mayor frecuencia y severidad ${ }^{(20)}$. Las infecciones recurrentes fueron muy frecuentes (92\%) en este estudio, sobre todo las del tracto respiratorio superior e inferior. De Moraes et al. ${ }^{(8)}$ reportaron que pacientes con asma presentan mayor susceptibilidad a infecciones respiratorias, principalmente las neumonías, lo cual también fue reportado recientemente ${ }^{(21)}$.

El principal objetivo de este estudio fue detectar casos de DPA y establecer su frecuencia en pacientes pediátricos con alergias, para ello, evaluamos los niveles séricos de IgA, IgG e IgM. En cuanto a la IgE, un $88 \%$ presentó valores elevados; un rasgo distintivo en la mayoría de los individuos atópicos es la elevación de los niveles de IgE, no obstante, existen personas con sintomatología altamente sugestiva de enfermedad alérgica, pero con niveles normales de esta inmunoglobulina ${ }^{(22)}$. Si bien la mayoría ( $83 \%$ ) de los pacientes alérgicos presentó niveles séricos normales de inmunoglobulinas totales, en un $17 \%$ (11/64) observamos hipogammaglobulinemia y cada uno de estos casos se clasificó en una DPA. Son escasos los reportes sobre la frecuencia de estas deficiencias en población pediátrica alérgica; Costa-
Carvalho et al. ${ }^{(10)}$ reportaron una frecuencia de $7 \%$ (2/27), De Moraes et al. ${ }^{(8)}$ una frecuencia de $61 \%$ (25/41) y Yuruker et al. ${ }^{(11)}$ reportaron 60,8\% (76/125). Estas diferencias podrían deberse a tamaños de muestras diferentes, a los métodos empleados para medir las inmunoglobulinas, a los criterios de diagnóstico fenotípico adoptados, e incluso, a la presencia de la deficiencia de subclases de IgG y/o la de anticuerpos específicos (SAD), las cuales no fueron abordadas en este estudio y se reportan con elevada prevalencia en niños alérgicos ${ }^{(8,9,23)}$.

La deficiencia de inmunoglobulina A (DIgA) fue la más frecuente; estudios previos también detectaron esta deficiencia ${ }^{(8,10)}$. Se describe a la DIgA como la IDP más común, con prevalencia aproximada de 1:600, donde $2 / 3$ de los afectados permanecen asintomáticos, mientras el $1 / 3$ sufre infecciones bacterianas recurrentes principalmente del tracto respiratorio, así como desórdenes gastrointestinales, autoinmunidad y enfermedad alérgica ${ }^{(13)}$. La falta o ausencia de la acción protectora de las mucosas por la IgA permite mayor penetración de los alérgenos, lo que induce la producción de IgE específica contra estos, dando como resultado la mayor prevalencia de enfermedades alérgicas en portadores de DIgA ${ }^{(24)}$. Es conveniente que los pacientes con DIgA sean seguidos en el tiempo, ya que podría progresar a inmunodeficiencia común variable (CVID), una deficiencia con fenotipo clínico y pronóstico más severos ${ }^{(25)}$.

Otra deficiencia de anticuerpos que encontramos fue la aislada de IgG. El algoritmo diagnóstico de $\mathrm{IUIS}^{(6)}$, clasifica a esta deficiencia dentro del grupo de las DPA con fenotipo CVID y propone que los bajos niveles de IgG pueden deberse a defectos en la proteína CD20 o CD21 de las células B. Por otra parte, los criterios diagnósticos de la AAAAI, la definen como una hipogammaglobulinemia no especificada, que podría incluso tener causas secundarias o ser transitoria de la infancia ${ }^{(15)}$, al respecto, debe considerarse que el tratamiento de las alergias con corticosteroides, principalmente del asma, pueden conducir a hipogammaglobulinemia de $\operatorname{Ig} G^{(26)}$. Estudios previos ${ }^{(8,10)}$ no reportaron esta deficiencia en su población de niños alérgicos, por tanto, este trabajo estaría aportando un nuevo hallazgo y resalta la importancia de considerar a la 
deficiencia aislada de $\operatorname{IgG}$ en población pediátrica alérgica, por su probable implicancia terapéutica ${ }^{(15)}$. Se encontró un solo caso de CVID, deficiencia que tampoco se reportó en estudios previos que evaluaron DPA en niños alérgicos ${ }^{(8,10)}$, a excepción del trabajo de Yuruker et al. ${ }^{(11)}$ donde observaron 7/125 casos de CVID en niños con asma. El diagnóstico de CVID es importante ya que se trata de una DPA con fenotipo clínico más severo y que precisa de tratamiento inmediato. En cuanto al tratamiento de pacientes con DPA, las estrategias normalmente quedan a decisión del médico especialista según la severidad y recurrencia de las infecciones. Una propuesta terapéutica indicada por expertos es la profilaxis antibiótica y/o terapia de reemplazo con IgG humana para CVID y XLA, deficiencias consideradas severas. Para DPA consideradas leves (DIgA, deficiencia de IgG aislada, deficiencia de subclases de IgG y SAD) la profilaxis antibiótica es la terapia más frecuente, sin embargo, en algunos casos es requerido el uso de terapia de reemplazo de $\operatorname{Ig} G^{(15)}$.

Finalmente, las características más resaltantes en el grupo de pacientes con DPA fueron las infecciones respiratorias recurrentes $(8 / 11)$ y el predominio $(9 / 11)$ de asma y rinitis alérgica. El espectro clínico de las DPA es muy amplio, siendo la característica clínica predominante la presencia de infecciones respiratorias recurrentes, principalmente las del tipo bacterianas, lo que resalta el rol de los anticuerpos en la protección de las mucosas ${ }^{2}$ Por otra parte, las deficiencias de anticuerpos se asocian a la presentación de enfermedad alérgica ya que la ausencia o los niveles reducidos de inmunoglobulinas IgA, IgG y/o IgM conducirían a que los alérgenos atraviesen las mucosas y aparezca la sensibilización, la producción de $\mathrm{IgE}$ específica y por último la reacción alérgica ${ }^{(21)}$.

Por último, no observamos diferencias significativas al comparar características clínico-demográficas entre los pacientes alérgicos con y sin DPA; esto nos conduce a pensar que probablemente no existiría un rasgo distintivo que pueda orientar al médico especialista a sospechar una DPA, por tanto, todo paciente pediátrico con enfermedad alérgica debería ser evaluado para deficiencias de anticuerpos.
Las principales limitaciones de este estudio fueron, por un lado, el reducido tamaño de muestra, lo que podría afectar la precisión de la frecuencia de DPA y las inferencias estadísticas presentadas; con la inclusión de más pacientes se lograría detectar un mayor número de casos y una mejor caracterización clínico-demográfica. Por otro lado, también fue un factor limitante no haber contemplado el estudio de la deficiencia de subclases de IgG y de anticuerpos específicos (SAD), ambas de elevada prevalencia en niños alérgicos, por tanto, nuestra frecuencia podría estar subestimada y los pacientes con inmunoglobulinas normales de esta serie podrían presentar dichas deficiencias.

\section{CONCLUSIONES}

En este estudio evaluamos los niveles séricos de IgA, IgG e IgM en pacientes pediátricos alérgicos y detectamos 11 casos de DPA, de los cuales 6 fueron DIgA, 4 deficiencias aisladas de IgG y un caso de CVID. La elevada frecuencia de DPA resalta la importancia de considerar el estudio de estas deficiencias en población pediátrica alérgica, de manera a lograr una detección temprana para un tratamiento oportuno, lo cual mejoraría la calidad de vida del paciente afectado y la de su familia.

Es la primera vez que se describen casos de DPA en población pediátrica alérgica de Paraguay $\mathrm{y}$, las deficiencias de anticuerpos detectadas en este estudio contribuyen al fortalecimiento del diagnóstico y el registro de inmunodeficiencias primarias en el país.

\section{CONTRIBUCIÓN DE AUTORÍA}

Cada uno de los autores colaboró y estuvo plenamente involucrado en todos los pasos del proceso de investigación, desde la concepción del tema y diseño del estudio, hasta la elaboración y revisión de la versión final del manuscrito. 


\section{REFERENCIAS}

1. Tangye SG, Al-Herz W, Bousfiha A, Chatila T, Cunningham-Rundles C, Etzioni A, et al. Human Inborn Errors of Immunity: 2019 Update on the Classification from the International Union of Immunological Societies Expert Committee. J Clin Immunol. 2020;40(1):24-64. doi: https://dx.doi.org/10.1007/s10875-019-00737-x

2. Rezaei N, Aghamohammadi A, Notarangelo LD. Primary Immunodeficiency Diseases. Definition, Diagnosis and Management. 2da ed. Berlin, Heidelberg: Springer; 2017.

3. Özcan C, Metin A, Erkoço?lu M, Kocabas C. Bronchial hyperreactivity in children with antibody deficiencies. Allergol Immunopathol (Madr). 2015;43(1):57-61. doi: https://dx.doi.org/10.1016/j.aller.2013.09.014

4. Mallol J, Crane J, von Mutius E, Odhiambo J, Keil U, Stewart A, et al. The International Study of Asthma and Allergies in Childhood (ISAAC) Phase Three: A global synthesis. Allergol Immunopathol (Madr). 2013;41(2):7385. doi: https://dx.doi.org/10.1016/j.aller.2012.03.001

5. Simon D. Recent advances in clinical allergy and immunology. Int Arch Allergy Immunol. 2018;177(4):32433. doi: https://dx.doi.org/10.1159/000494931

6. Shkalim V, Monselize Y, Segal N, Zan-Bar I, Hoffer V, Garty B. Selective IgA deficiency in children in Israel. J Clin Immunol. 2010;30(5):761-5. doi: https://dx.doi.org/ 10.1007/s10875-010-9438-x

7. Ramírez N, Arablin S, Mojica D, Yamazaki A, García M, Terán L, et al. Clinical and immunological features of common variable immunodeficiency in mexican patients. Allergol Immunopathol (Madr). 2014;42(3):235-40. doi: https://dx.doi.org/10.1016/j.aller.2013.01.007

8. De Moraes Lui C, Oliveira LC, Diogo CL, Kirschfink M, Grumachi AS. Immunoglobulin G subclass concentrations and infections in children and adolescents with severe asthma. Pediatr Allergy Immunol. 2002;13(3):195-202. doi: https://dx.doi.org/10.1034/j.1399-3038.2002.00058.x

9. Pérez González JM, Franco M. Niveles de subclases de IgG en la patología alérgica respiratoria infantil. Allergol Immunopathol (Madr). 2003;31(1):18-30.

10. Costa Carvalho BT, Nagao AT, Arslanian C, Carneiro $\mathrm{Mm}$, Naspitz $\mathrm{Ck}$, Sorensen $\mathrm{RU}$, et al. Immunological evaluation of allergic respiratory children with recurrent sinusitis. Pediatr Allergy Immunol. 2005; 16(6):534-8. doi: https://dx.doi.org/10.1111/j.1399-3038.2005.00303.x
11. Yuruker O, Sanlidag B, Uncu M, Bahceciler N. The role of hypogammaglobulinemia in childhood asthma. Iran Red Crescent Med J. 2018;20(9). doi: https://dx.doi.or g/10.5812/ircmj.62984

12. Caballero F, Benegas S, Giménez V, Granado E, Martínez C, Carpinelli MM, et al. Antibody deficiencies in children and adolescents with recurrent and/or severe infections. Pediatr (Asunción). 2018;45(2):141-6. doi: https://dx.doi.org/10.31698/ped.45022018006

13. Yel L. Selective IgA deficiency. J Clin Dermatology. 2012; 41(4):211-3. doi: https://dx.doi.org/10.1007/s10875-009-9357-x

14. Bousfiha A, Jeddane L, Picard C, Al-Herz W, Ailal F, Chatila T, et al. Human Inborn Errors of Immunity: 2019 Update of the IUIS Phenotypical Classification. J Clin Immunol. 2020;40(1):66-81. doi: 10.1007/s10875-020-00758-x

15. Bonilla FA, Khan DA, Ballas ZK, Chinen J, Frank MM, Hsu JT, et al. Practice parameter for the diagnosis and management of primary immunodeficiency. J Allergy Clin Immunol. 2014; 136(5):1186-1205.e78. doi: https://dx.doi.org/10.1016/j.jaci.2015.04.049

16. Ha EK, Na MS, Lee S, BAek H, Lee S, Sheen Y, et al. Prevalence and Clinical Characteristics of Local Allergic Rhinitis in Children Sensitized to House Dust Mites. Int Arch Allergy Immunol. 2017; 174:e62984. doi: https://dx.doi.org/10.1159/000481091

17. Dharmage SC, Perret JL, Custovic A. Epidemiology of asthma in children and adults. Front Pediatr. 2019; 7(246):115. doi: https://dx.doi.org/10.3389/fped.2019.00246

18. Bosnic-AnticevichS, Smith P, Abramson M, Hespe CM, Johnson M, Stosic R, et al. Impact of allergic rhinitis on the day-to-day lives of children: insights from an Australian cross-sectional study. BMJ Open. 2020; 10:e038870. doi: https://dx.doi.org/10.1136/bmjopen-2020-038870

19. Del-rio-navarro B, Ito-Tsuchiya F, Zepeda B. Rinitis, sinusitis y alergia. Rev Alerg México. 2015;56(6):204-16.

20. Rodríguez-González M, Espinosa-Rosales F. Uso de glucocorticoides en enfermedades alérgicas. Acta Pediatr Mex. 2017;38(1):63-71. doi: https://dx.doi.org/10.18233/ap m1no1pp63-711324

21. Zaidi SR, Blakey JD. Why are people with asthma susceptible to pneumonia? A review of factors related to upper airway bacteria. Respirology. 2019; 24(5):423-30. doi https://dx.doi.org/10.1111/resp.13528 
22. Baillieau F. Inmunoglobulina E: Revisión y actualización de su rol en la salud y enfermedad. Arch Alerg e Inmunol Clin. 2015; 46(2):54-66.

23. Quezada A, Norambuena X, Inostroza J, Rodríguez J. Specific antibody deficiency with normal immunoglobulin concentration in children with recurrent respiratory infections. Allergol Immunopathol (Madr). 2015; 43(3):2927. doi: https://dx.doi.org/10.1016/j.aller.2014.07.009

24. De Oliveira-Serra FA, Mosca T, Da Conceição Santos De Menezes M, Carvalho-Neves W. Clinical symptoms in IgA deficiency. Rev Alerg Mex. 2017;64(1):34-9. doi: https://dx.doi.org/10.29262/ram.v64i1.216
25. Aghamohammadi A, Mohammadi J, Parvaneh N, Rezaei N, Moin M, Espanol T, et al. Progression of selective IgA deficiency to common variable immunodeficiency. Int Arch Allergy Immunol. 2008;147(2):87-92. doi: https://dx.doi.org/10.1159/000135694

26. Wirsum C, Glaser C, Gutenberger S, Keller B, Unger S, Voll RE, et al. Secondary antibody deficiency in glucocorticoid therapy clearly differs from primary antibody deficiency. J Clin Immunol. 2016; 36:406-12. doi: https://dx.doi.org/10.1007/s10875-016-0264-7 